Audit report

Pre-publication: accepted (01.08.2019) - (final amended) version

International Journal of STD \& AIDS 2019

Article reuse guidelines: sagepub.com/journals-permissions DOI:

$10.1177 / 0956462419871857$ journals.sagepub.com/home/std

\title{
Behind the camera: Sexual health testing patterns and outcomes amongst UK adult film performers
}

Gregory Patrick King ${ }^{1}$ and David Thomas Evans ${ }^{2}$

\begin{abstract}
We report the frequency of sexually transmitted infections (STIS) diagnosed in UK adult film industry performers. A total of 100 adult film performers regularly screened for STIs on an average of every three weeks. High rates of condomless sex were reported and there were STIs recorded during the 19-year period covered by this study (1996-2015). STIs recorded included gonorrhoea, chlamydia, non-gonococcal urethritis, genital warts, syphilis and Trichomonas vaginalis.
\end{abstract}

\section{Keywords}

Sexually transmitted infections, adult film industry, sexual behaviour, safer sex, post exposure prophylaxis

Date received: 28 April 2019; accepted: 1 August 2019

\section{Background}

Some UK Adult Film Industry (AFI) employers recommend performers to have regular sexually transmitted infection (STI) screening, prior to filming, and require a 'negative' test/certificate. ${ }^{1}$ Adult film performers (AFPs) who have screening may regard testing as a harm reduction strategy; screening does not necessarily prevent sexual infections but may serve as an early warning system in relation to specific infections, e.g. human immunodeficiency virus (HIV), syphilis, gonorrhoea and chlamydia. Research in USA and UK on AFPs report that sexual intercourse is condomless $80 \%-90 \%$ of the time, ${ }^{2-7}$ and the prevalence of STI is between $8 \%$ and $35 \%$.

\footnotetext{
1East Sussex Healthcare NHS Trust, Hastings, UK

2Faculty of Education and Health, University of Greenwich, London, UK

Corresponding author:

Gregory Patrick King, East Sussex Healthcare NHS Trust, Station Plaza

Health Centre, 1st Floor, Station Plaza, Hastings, TN37 7RD, UK. Email: greg.king@nhs.net
} 


\section{Aim}

The aim of this study is to ascertain the frequency of AFPs' STI testing in the UK, in relation to infection diagnoses and sequalae.

\section{Methods}

Two audits were undertaken, by a sole master's degree researcher, of 100 self-identified AFPs' case notes at an inner London, England, specialist sexual health service for sex workers. The audits covered 2014-2015 (Audit 1) and 1996-2015 (Audit 2).

In Audit 1, information was gathered on sexual orientation, sexual practices, frequency of attendance at clinic and prevalence of condomless sex in a single year. This selected time period in Audit 1 gave an up to date and detailed representation of consultations with GU physicians and nurses, in response to Adult Film Industry (AFI) recommendations.

Audit 2 was the first in the UK to assess the prevalence of STIs among AFPs attending this clinic over a substantial period of time, 1996 to 2015.

\section{Results}

Selected results from Audit 1 show that between 2014 and 2015, 38\% $(n=38)$ of AFPs attended testing every three weeks, whereas two APFs choose weekly testing. There was a high occurrence of condomless sex acts by AFPs: $85 \%(n=530)$ compared to $15 \%(n=94)$ intercourse with condom (Table 1). The percentage of condomless sex in the female AFPs' professional work decreased slightly when compared to their personal lives (81\% compared with $85 \%$ ), whereas in male AFPs, the percentage decreased from $84 \%$ in personal life to $91 \%$ in professional practice. Females were diverse in sex activities for work, including $16 \%$ $(n=8)$ performing oro-anal sex ('rimming'), 24\% ( $n=12)$ mutual masturbation or 'fingering' and $38 \%(n=19)$ having received anal intercourse (Table 1$)$.

Table 1: Demographics and sexual practices among the 100 AFPs 2014 to 2015.

\begin{tabular}{|c|c|c|c|c|}
\hline \multirow[b]{2}{*}{ Age (years) } & \multicolumn{2}{|c|}{$\begin{array}{l}\text { Male Adult Film Performers } \\
\qquad(n=50)\end{array}$} & \multicolumn{2}{|c|}{$\begin{array}{l}\text { Female Adult Film Performers } \\
\qquad(n=50)\end{array}$} \\
\hline & $\mathrm{n}$ & $\%$ & $\mathrm{n}$ & $\%$ \\
\hline $18-30$ & 9 & 18 & 19 & 38 \\
\hline $31-39$ & 16 & 32 & 14 & 28 \\
\hline $40-49$ & 12 & 24 & 5 & 10 \\
\hline$>50$ & 13 & 26 & 12 & 24 \\
\hline Median age & 39 & & 32 & \\
\hline Sexual Orientation & Private & Professional & Private & Professional \\
\hline Heterosexual & 42 & 45 & 42 & 37 \\
\hline Homosexual & 3 & 4 & 1 & 1 \\
\hline Not Specified & 3 & 0 & 4 & 6 \\
\hline Bisexual & 2 & 1 & 3 & 6 \\
\hline Transgender & 0 & 0 & 0 & 0 \\
\hline
\end{tabular}




\begin{tabular}{|c|c|c|c|c|c|c|c|c|}
\hline \multirow[t]{2}{*}{ Sexual Practice } & \multicolumn{2}{|c|}{ With Condom } & \multicolumn{2}{|c|}{ Condomless } & \multicolumn{2}{|c|}{ With Condom } & \multicolumn{2}{|c|}{ Condomless } \\
\hline & $\mathrm{n}$ & $\%$ & $\mathrm{n}$ & $\%$ & $\mathrm{n}$ & $\%$ & $\mathrm{n}$ & $\%$ \\
\hline \multicolumn{9}{|l|}{ Personal } \\
\hline Oral & 3 & 5 & 52 & 95 & 4 & 5 & 69 & 95 \\
\hline Vaginal & 18 & 29 & 45 & 71 & 14 & 19 & 60 & 81 \\
\hline Anal & 0 & 0 & 11 & 100 & 7 & 27 & 19 & 73 \\
\hline Mutual Masturbation & 0 & 0 & 0 & 0 & 2 & 40 & 3 & 60 \\
\hline Oro-Anal & 0 & 0 & 0 & 0 & 0 & 0 & 1 & 100 \\
\hline Total & 21 & 16 & 108 & 84 & 27 & 15 & 152 & 85 \\
\hline \multicolumn{9}{|l|}{ Professional } \\
\hline Oral & 2 & 3 & 66 & 97 & 22 & 25 & 66 & 75 \\
\hline Vaginal & 7 & 13 & 48 & 87 & 6 & 12 & 44 & 88 \\
\hline Anal & 4 & 29 & 10 & 71 & 3 & 16 & 16 & 84 \\
\hline Mutual Masturbation & 0 & 0 & 0 & 0 & 1 & 8 & 11 & 92 \\
\hline Oro-Anal & 0 & 0 & 0 & 0 & 1 & 13 & 7 & 87 \\
\hline Toys & 0 & 0 & 2 & 100 & 0 & 0 & 0 & 0 \\
\hline Total & 13 & 9 & 126 & 91 & 33 & 19 & 144 & 81 \\
\hline Combined Total & 34 & 13 & 234 & 87 & 60 & 17 & 296 & 83 \\
\hline
\end{tabular}

Twenty-one female AFPs worked in other areas of sex work: 17 as escorts, 2 in escorting / webcamming and 2 worked at sex parties / swingers' clubs. Two males worked as escorts. In Audit 2, 171 new diagnoses of STIs were recorded from 1996 to 2015 (genital warts ( $n=31)$, gonorrhoea $(n=40)$, non-gonococcal urethritis $(n=41)$ chlamydia $(n=35)$, Trichomonas vaginalis $(n=1)$ and syphilis $(n=1)$ ) (Table 2$)$. There were no cases of HIV, hepatitis B or hepatitis C. Males were more likely to have an STI than females: 98 compared to 73 . Hepatitis vaccination uptake showed 34 of the patients commenced hepatitis $A$ and 77 commenced hepatitis $B$ vaccination.

\section{Discussion}

UK AFPs demonstrate a preference for testing every 28 days (a self-determined AFI UK standard $)^{8}$; however, US researchers recommended testing 7-14 days in order to minimise STIs. ${ }^{7,9}$ The US Free Speech Coalition responded to this shorter timeframe and introduced the Performer Availability Screening Services 'Moratorium' which recommends terminating filming if HIV is detected. ${ }^{10}$ No similar system exists in the UK and it is questionable how effective this policy is at preventing STIS/HIV, as no robust evidence exists.

Our study highlighted females do not exclusively work in one genre of sex work, and further research utilising tools aimed at HIV prevention (condom use, Treatment as Prevention (TasP), Post Exposure Prophylaxis (PEP), (Pre-Exposure Prophylaxis (PrEP) and Undetectable equals Uninfectious $(U=U)$ may be fruitful in the future. The study was conducted retrospectively and was limited due to reliance on presumed accurate documentation. An under-estimation of the prevalence of STIs in this cohort is possible, as online testing and treatment of STIs are now readily available, and/or the cohort could attend other National Health Service / private clinics. 
Table 2. Sexual infections in 100 AFPs audit by gender and sexuality (1996 to 2015).

\begin{tabular}{|c|c|c|c|c|c|c|c|c|c|c|c|c|c|c|c|c|c|c|}
\hline & \multicolumn{8}{|c|}{ Male AFPs } & \multicolumn{8}{|c|}{ Female AFPs } & \multirow{2}{*}{\multicolumn{2}{|c|}{$\begin{array}{l}\text { All AFPs } \\
\text { All Groups }\end{array}$}} \\
\hline & \multicolumn{2}{|c|}{ Bisexual } & \multicolumn{2}{|c|}{ Heterosexual } & \multicolumn{2}{|c|}{ Homosexual } & \multicolumn{2}{|c|}{ All Groups } & \multicolumn{2}{|c|}{ Bisexual } & \multicolumn{2}{|c|}{ Heterosexual } & \multicolumn{2}{|c|}{ Not specified } & \multicolumn{2}{|c|}{ All Groups } & & \\
\hline & $\begin{array}{l}\% \text { of } \\
\text { total } \\
\text { STIs }\end{array}$ & $\begin{array}{l}\text { Diagnosed } \\
\text { (n) }\end{array}$ & $\begin{array}{l}\% \text { of } \\
\text { total } \\
\text { STIs }\end{array}$ & $\begin{array}{l}\text { Diagnosed } \\
\text { (n) }\end{array}$ & $\begin{array}{l}\% \text { of } \\
\text { total } \\
\text { STIs }\end{array}$ & $\begin{array}{l}\text { Diagnosed } \\
\text { (n) }\end{array}$ & $\begin{array}{l}\% \text { of } \\
\text { total } \\
\text { STIs }\end{array}$ & $\begin{array}{l}\text { Diagnosed } \\
\text { (n) }\end{array}$ & $\begin{array}{l}\% \text { of } \\
\text { total } \\
\text { STIs }\end{array}$ & $\begin{array}{l}\text { Diagnosed } \\
\text { (n) }\end{array}$ & $\begin{array}{l}\% \text { of } \\
\text { total } \\
\text { STIs }\end{array}$ & $\begin{array}{l}\text { Diagnosed } \\
\text { (n) }\end{array}$ & $\begin{array}{l}\% \text { of } \\
\text { total } \\
\text { STIs }\end{array}$ & $\begin{array}{l}\text { Diagnosed } \\
\text { (n) }\end{array}$ & $\begin{array}{l}\% \text { of } \\
\text { total } \\
\text { STIs }\end{array}$ & $\begin{array}{l}\text { Diagnosed } \\
\text { (n) }\end{array}$ & $\begin{array}{l}\% \text { of } \\
\text { total } \\
\text { STIs }\end{array}$ & $\begin{array}{l}\text { Diagnosed } \\
\text { (n) }\end{array}$ \\
\hline Non-gonococcal urethritis & 20 & 4 & 28 & 27 & 21 & 3 & 26 & 34 & 4 & 1 & 3 & 6 & 0 & 0 & 3 & 7 & 11 & 41 \\
\hline Gonorrhoea-male-urethral & 0 & 0 & 3 & 3 & 0 & 0 & 2 & 3 & $n / a$ & & & & & & & & $\mathrm{n} / \mathrm{a}$ & \\
\hline Gonorrhoea-rectal & 5 & 1 & 0 & 0 & 7 & 1 & 2 & 2 & 0 & 0 & 3 & 6 & 0 & 0 & 2 & 6 & 2 & 8 \\
\hline Gonorrhoea-suspected & 0 & 0 & 0 & 0 & 0 & 0 & 0 & 0 & 0 & 0 & 2 & 5 & 0 & 0 & 2 & 5 & 1 & 5 \\
\hline Gonorrhoea-oropharyngeal & 15 & 3 & 5 & 5 & 0 & 0 & 6 & 8 & 0 & 0 & 3 & 7 & 0 & 0 & 3 & 7 & 4 & 15 \\
\hline Gonorrhoea-uncomplicated & 5 & 1 & 3 & 3 & 0 & 0 & 3 & 4 & 0 & 0 & 2 & 5 & 0 & 0 & 2 & 5 & 2 & 9 \\
\hline Chlamydia & 10 & 2 & 9 & 9 & 7 & 1 & 9 & 12 & 4 & 1 & 5 & 11 & 25 & 1 & 5 & 13 & 7 & 25 \\
\hline Chlamydia-rectal & 0 & 0 & 0 & 0 & 14 & 2 & 2 & 2 & 0 & 0 & 0 & 0 & 0 & 0 & 0 & 0 & 1 & 2 \\
\hline Chlamydia-suspected & 0 & 0 & 3 & 3 & 7 & 1 & 3 & 4 & 0 & 0 & 0 & 1 & 0 & 0 & 0 & 1 & 1 & 5 \\
\hline Chlamydia-pharyngeal & 15 & 3 & 0 & 0 & 0 & 0 & 2 & 3 & 0 & 0 & 0 & 0 & 0 & 0 & 0 & 0 & 1 & 3 \\
\hline Gonorrhaea-cervical & $n / a$ & & & & & & & & 0 & 0 & 2 & 4 & 0 & 0 & 2 & 4 & $\mathrm{n} / \mathrm{a}$ & \\
\hline Genital warts-first occurrence & 0 & 0 & 9 & 9 & 29 & 4 & 10 & 13 & 0 & 0 & 5 & 11 & 0 & 0 & 4 & 11 & 8 & 31 \\
\hline Genital warts-recurrence & 20 & 4 & 15 & 14 & 14 & 2 & 15 & 20 & 0 & 0 & 2 & 4 & 50 & 2 & 2 & 6 & 5 & 19 \\
\hline Molluscumcontagiosum & 0 & 0 & 8 & 8 & 0 & 0 & 6 & 8 & 0 & 0 & 2 & 4 & 0 & 0 & 2 & 4 & 3 & 12 \\
\hline Pelvic inflammatory disease & $n / a$ & & & & & & & & 4 & 1 & 3 & 6 & 0 & 0 & 3 & 7 & $\mathrm{n} / \mathrm{a}$ & \\
\hline Genital herpes-first episode & 5 & 1 & 4 & 4 & 0 & 0 & 4 & 5 & 0 & 0 & 0 & 1 & 0 & 0 & 0 & 1 & 2 & 6 \\
\hline Genital herpes-recurrence & 0 & 0 & 0 & 0 & 0 & 0 & 0 & 0 & 0 & 0 & 2 & 4 & 0 & 0 & 2 & 4 & 1 & 4 \\
\hline Syphilis & 0 & 0 & 0 & 0 & 0 & 0 & 0 & 0 & 0 & 0 & 0 & 1 & 0 & 0 & 0 & 1 & 0 & 1 \\
\hline Trichomonas vaginalis & $\mathrm{n} / \mathrm{a}$ & & & & & & & & 0 & 0 & 0 & 1 & 0 & 0 & 0 & 1 & $\mathrm{n} / \mathrm{a}$ & \\
\hline Total & & 19 & & 85 & & 14 & & 118 & & 3 & & 77 & & 3 & & 83 & & 186 \\
\hline
\end{tabular}

AFP: adult film performer; STI: sexually transmitted infection. 
An unexpected finding identified gonorrhoea in five self-identified heterosexual men's pharynxes. This may support recommendations for three-site anatomical testing for gonorrhoea and chlamydia to reduce a possible reservoir of missed infections. ${ }^{4}$

To conclude, a challenging interplay exists between performers, healthcare providers and film producers in attaining and improving sexual health among these sex workers. To address any deficits in optimum service provision, there is a need to develop specific UK national guidance for clinicians, including improved collaboration with established AFI organisations such as United Kingdom Adult Producers and 'PORN4PREP'. It will also be important to undertake a proper review of the industry's occupational safety mechanisms for performers and to question AFPs' personal risk reduction strategies.

\section{Declaration of conflicting interests}

The authors declared no potential conflicts of interest with respect to the research, authorship, and / or publication of this article.

\section{Funding}

The authors disclosed receipt of the following financial support for the research, authorship, and/or publication of this article: This research was supported by Imperial College Healthcare NHS Trust towards Master of Arts degree in Professional practice (Sexual health Route).

\section{ORCID iD}

Gregory Patrick King https://orcid.org/0000-0002-9706-4945

David Thomas Evans https://orcid.org/0000-0001-6874-3845

\section{References}

1. Grudzen CR and Kerndt PR. The adult film industry: time to regulate? PLoS Med 2007; 4: e126 (2007, accessed 02 February 2019).

2. Javanbakht $M$, Gorbach $\mathrm{P}$, Dillavou $\mathrm{C}$, et al. Adult film performers transmission behaviors and STI prevalence. In: National STD prevention conference (Sexually transmitted infections, Supplement 1), Atlanta, GA, 1 June 2014.

3. Taylor MM, Rotblatt $\mathrm{H}$, Brooks JT, et al. Epidemiologic investigation of a cluster of workplace HIV infections in the adult film industry: Los Angeles, California, 2004. Clin Infect Dis 2007; 44: 301-305.

4. Coyne KM, Banks A, Heggie $C$, et al. Sexual health of adults working in pornographic films. Int J Std Aids 2009; 20: 508-509.

5. Hill SC, King G and Smith A. Condom use and prevalence of sexually transmitted infection among performers in the adult entertainment industry. Int J Std Aids 2009; 20: 809-810.

6. Goldstein BY, Steinberg JK, Aynalem G, et al. High Chlamydia and Gonorrhea incidence and reinfection among performers in the adult film industry. Sex Transm Dis 2011; 38: 644-648.

7. Rodriguez-Hart C, Chitale RA, Rigg R, et al. Sexually transmitted infection testing of adult film performers: is disease being missed? Sex Transm Dis 2012; 39: 989-994. 
8. Gabrielsen LL and Barton SE. HIV negative certification and sexual health issues facing performers in the adult entertainment industry in the UK. Sex Transm Infect 2002; 78: 311.

9. Javanbakht M, Dillavou MC, Rigg RW Jr, et al. Transmission behaviors and prevalence of Chlamydia and Gonorrhea among adult film performers. Sex Transm Dis 2017; 44: 181-186.

10. Free Speech Coalition. An FAQ about STIs, testing and moratoriums, https://www.freespeechcoalition.com/blog/2013/12/08/an-faq-about-stis-testingand-moratoriums (2013, accessed 10 February 2019). 